\title{
ALTA FRECUENCIA DE PLAGIO EN TESIS DE MEDICINA DE UNA UNIVERSIDAD PÚBLICA PERUANA
}

\author{
J. Jhan C. Saldaña-Gastulo, ${ }^{1,2 a}$, C. Claudia Quezada-Osoriaa,b, Américo Peña-Oscuvilca ${ }^{2,3 a}$, \\ Percy Mayta-Tristán ${ }^{2,4,5, c}$
}

\begin{abstract}
RESUMEN
Se realizó un estudio observacional con el objetivo de describir la presencia de plagio en tesis de medicina ejecutadas en el año 2008 en una universidad pública peruana. Se buscó plagio en las introducciones de 33 tesis usando un algoritmo de búsqueda con Google, se caracterizó el tipo de estudio y se buscó en forma electrónica si los asesores de las tesis han publicado artículos en revistas científicas. Se encontró evidencia de plagio en $27 / 33$ de las introducciones, 37,3\% (171/479) de todos los párrafos analizados tuvieron algún grado de plagio, el plagio literal fue el más frecuente (20/27) y las fuentes de plagio más comunes fueron revistas científicas (19/27). Las características de los estudios analizados fueron observacionales (32/33), transversales (30/33), descriptivos (25/33) y retrospectivos (19/33). Ninguno de los autores había publicado en una revista científica, y sólo nueve de sus asesores de ellos tenían al menos una publicación. No se encontró asociación entre las características de la tesis y la presencia de plagio. En conclusión, se encontró una alta frecuencia de plagio en las tesis analizadas. Es responsabilidad de las facultades de medicina tomar las medidas necesarias para detectar y evitar el plagio entre sus estudiantes.
\end{abstract}

Palabras clave: Tesis Académicas; Plagio; Mala conducta científica; Educación médica; Perú (fuente: DeCS BIREME).

\section{HIGH FREQUENCY OF PLAGIARISM IN MEDICAL THESIS FROM A PERUVIAN PUBLIC UNIVERSITY}

\begin{abstract}
An observational study was conducted to describe the presence of plagiarism in medical thesis in 2008 performed at a public university in Peru. Search for plagiarism in 33 thesis introductions using a Google search algorithm, characterizes of the study type and we search in electronic form if the thesis mentor have published articles in scientific journals. We found evidence of plagiarism in $27 / 33$ introductions, $37.3 \%$ (171/479) of all the paragraphs analyzed had some degree of plagiarism, literal plagiarism was the most frequent (20/27) and journals were the most common sources of plagiarism (19/27). The characteristics of the studies were observational (32/33), cross-sectional (30/33), descriptive (25/33) and retrospective (19/33). None of the authors had published in a scientific journal, and only nine of his tutors of them had at least one publication. No association was found between the characteristics of the thesis and the presence of plagiarism. In conclusion, we found a high frequency of plagiarism in theses analyzed. Is responsibility of medical schools take the necessary actions to detect and avoid plagiarism among their students.
\end{abstract}

Key words: Academic dissertations; Plagiarism; Scientific misconduct; Medical education; Peru (source: MeSH NLM).

\section{INTRODUCCIÓN}

La tesis es el producto de una investigación original que un estudiante desarrolla para optar por un título o grado académico universitario. En el Perú, resulta ser una modalidad de graduación voluntaria en diferentes facultades de medicina, situación que ha generado que los graduados por esta vía sean escasos en estos centros de estudios superiores (1), no obstante, existen universidades que la tienen como requisito obligatorio; en ambos casos existen estudiantes sin interés por la investigación ${ }^{(2)}$, con la posibilidad que éstos inventen, manipulen o plagien información, como se ha observado en algunas instituciones académicas ${ }^{(3,4)}$.

\footnotetext{
Sociedad Científica de Estudiantes de Medicina de la Universidad Nacional de Piura, Facultad de Medicina Humana, Universidad Nacional de Piura. Piura, Perú.

2 Sociedad Científica Médico Estudiantil Peruana, Perú.

3 Sociedad Científica Huachana de Estudiantes de Medicina, Facultad de Medicina Humana, Universidad Nacional José Faustino Sánchez Carrión. Huacho, Perú.

4 Oficina General de Información y Sistemas, Instituto Nacional de Salud. Lima, Perú.

5 Escuela de Medicina, Universidad Peruana de Ciencias Aplicadas. Lima, Perú

a Interno de Medicina; ${ }^{\text {b }}$ Alumna de Medicina; ${ }^{\text {c Médico. }}$
}

Recibido: 01-02-10 Aprobado: 17-03-10 
Se define plagio a la apropiación de las ideas, procesos o resultados, presentados en una publicación sin dar crédito al autor de la misma ${ }^{(5,6)}$. Hoy en día, el uso de medios informáticos, podría facilitar que el plagio se cometa, debido a que la información está disponible en Internet y se cuenta con procesadores de textos que permiten "copiar y pegar" escritos (7). Varios estudios han mostrado que este tipo de fraude es frecuente en la elaboración de las tesis y trabajos de investigación ${ }^{(7-10)}$.

Así como los recursos electrónicos lo posibilitan, también podrían ser una herramienta útil para su detección; como el caso de los motores de búsqueda de Google (http:// www.google.com) y Google académico (www.scholar. google.com) ${ }^{(4,8)}$.

El objetivo del estudio fue determinar la frecuencia de plagio en tesis de medicina de una universidad pública peruana.

\section{EL ESTUDIO}

Se realizó un estudio observacional y transversal durante los meses de abril a junio del 2009 que incluyó el total de las tesis sustentadas para optar el Título de Médico Cirujano durante el año 2008 en una universidad pública peruana, donde su ejecución individual es la única forma de graduación. El estudio fue autorizado por el Comité de Investigación de la Facultad de Medicina de esta universidad y aprobado por el Comité Institucional de Ética e Investigación del Instituto Nacional de Salud (Reg. 20304-09).

Se obtuvo una copia simple del apartado correspondiente a introducción y referencias bibliográficas de la tesis, en una ficha se recolectó los nombres del autor y asesor, el área de estudio (salud pública y educación médica, ciencias básicas o clínico quirúrgicas), características del estudio según la intervención del autor (observacional o experimental), número de mediciones (transversal o longitudinal), la temporalidad (prospectivo o retrospectivo), la existencia de comparación (descriptivo o analítico) y tipo de estadística usada (descriptiva o inferencial) ${ }^{(12)}$.

Para determinar la presencia de publicaciones de autores y asesores se usó el motor de búsqueda de Google Académico siguiendo el algoritmo de búsqueda propuesto por Mayta-Tristán y Mezones-Holguín (13), y en caso de encontrarse publicaciones del autor o del asesor, se procedió a comprobar si una de ellas era la tesis correspondiente.

La presencia de plagio se determinó ingresando individualmente los párrafos del apartado de Introducción en el motor de búsqueda de Google, con la finalidad de identificar textos cuyo contenido fuese similar o igual al del párrafo. De no encontrarse resultados similares en esta búsqueda, se procedía a realizó un segundo intento eliminando conectores y ciertas palabras al azar, tal como se ha realizado en estudios previos ${ }^{(8,9)}$.

De encontrarse párrafos con presencia de plagio, se procedió a determinar si este era literal (todo el párrafo era copiado) o parcial (algunas partes eran iguales, pero algunos conectores o frases eran diferentes) y si la fuente de plagio era de contenido científico técnico (revista indizada, texto o literatura médica en línea) o páginas web de información general no especializada ${ }^{(9)}$.

Asimismo, se calculó la proporción de plagio, definida como el cociente entre el número de párrafos con evidencia de plagio sobre el número de párrafos totales de la introducción multiplicado por 100 , esto basado y adaptado de las publicaciones de Billic-Zulle L et al. ${ }^{(3)} \mathrm{y}$ Huamaní C et al. ${ }^{(9)}$.

Se elaboró una base de datos en el paquete estadístico SPSS versión 15.0. Se evaluó la asociación entre la presencia de plagio con el tipo de estudio o la publicación de artículos por parte del asesor, para ello se usó la prueba exacta de Fisher y se calculó el OR. Se consideró un $p<0,05$ como significativo.

\section{HALLAZGOS}

Se incluyó 33 tesis que contenían 459 párrafos en la sección de introducción, con una media de 13,9 \pm 6,2 párrafos por tesis. En 20/33 tesis los estudios fueron del área clínico quirúrgicas, 12 sobre salud pública y educación médica y solo 1 sobre ciencias básicas. Los tipos de estudio empleados fueron el observacional (32/33), retrospectivo (19/33), transversal (30/33) y descriptivo (25/33). El tipo de estadística más frecuentemente usada fue la descriptiva (18/33).

En cuanto a las publicaciones realizadas, ningún autor había publicado en una revista científica durante el pregrado, además, la tesis no había sido publicada hasta junio de 2009. Asimismo, sólo nueve asesores tenían al menos una publicación en una revista científica nacional, no se encontraron publicaciones internacionales.

El análisis de la introducción, determinó que existe evidencia de plagio en $171(37,3 \%)$ párrafos, que corresponden a 27 tesis (27/33), en estas el tipo de plagio más frecuente usado fue el literal (20/27) y las fuentes de plagio fueron en su mayoría revistas científicas (19/27). 


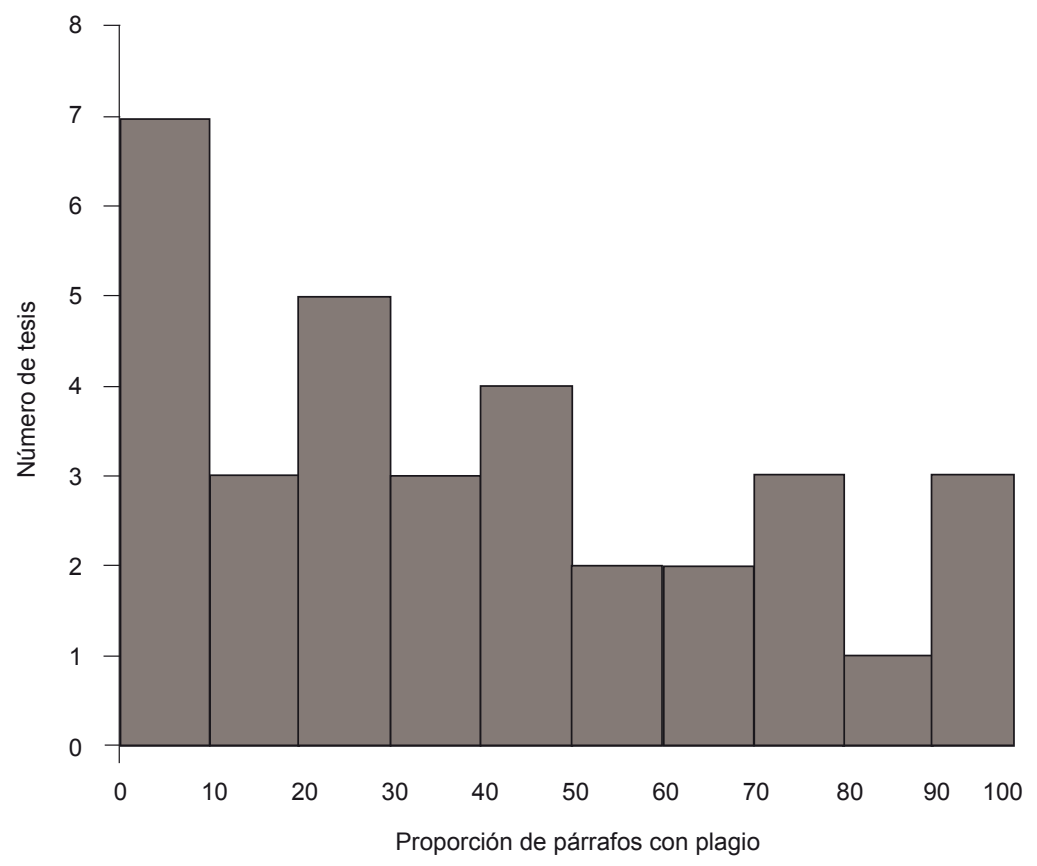

Figura 1. Proporción de plagio en las tesis de medicina sustentadas durante el 2008 en una universidad pública peruana. $\mathrm{N}=33$

La proporción de plagio media fue de $37,8 \% \pm 30,7 \%$, teniendo como valor máximo $92,3 \%$. Un total de 10 tesis presentaron una proporción de plagio menor del $20 \%$ y de estas 6 no tuvieron evidencia de plagio.

En el análisis bivariado no se encontró asociación entre la presencia de plagio y las distintas variables del estudio (Tabla 3); sin embargo, es posible que con una mayor muestra se pueda corroborar que las tesis bajo una asesoría con profesionales que publiquen, presentan con menos frecuencia plagio (OR: 0,$28 ; p=0,187$ ).

\section{DISCUSIÓN}

Nosotros encontramos que el plagio es frecuente en las tesis para optar el título de médico cirujano en una universidad peruana, sólo $6 / 33$ no tenían evidencia de plagio. Estos hallazgos son similares a estudios realizados sobre trabajos de investigación hechos por estudiantes del segundo año de medicina de otra universidad peruana, donde 23/24 tuvieron evidencia de plagio con el mismo sistema que hemos usado ${ }^{9}$. Por otro lado, en Croacia encontraron que sólo el $9 \%$ de los estudiantes de medicina no realiza plagio en sus trabajos académicos (3) y en EEUU encontraron que $27,3 \%$ de las 210 tesis de maestría analizadas tenían indicios de plagio ${ }^{(4)}$.

El tipo de plagio más frecuente en nuestro trabajo fue el tipo literal (20/33) que es mayor, al informado en estudiantes de medicina en otra universidad peruana $(8 / 23)^{(9)}$. Esto podría suceder porque hay un desconocimiento de éste como problema ético en los

Tabla 1. Factores evaluados con la presencia de plagio en las tesis desarrolladas durante el 2008 en una universidad pública peruana.

\begin{tabular}{|c|c|c|c|c|}
\hline \multirow{2}{*}{ Variable } & \multicolumn{2}{|c|}{ Plagio } & \multirow{2}{*}{ OR } & \multirow{2}{*}{$\mathbf{p}$} \\
\hline & Sí & No & & \\
\hline Tipo de estudio transversal & 25 & 5 & 2,50 & 0,464 \\
\hline Tipo de estudio retrospectivo & 16 & 3 & 1,45 & 0,510 \\
\hline Uso único de estadística descriptiva & 15 & 3 & 1,25 & 0,577 \\
\hline Tesis en el área de ciencias clínicas & 16 & 4 & 0,72 & 0,558 \\
\hline Número de párrafos mayor de 13 & 15 & 4 & 0,62 & 0,489 \\
\hline Tipo de estudio descriptivo & 20 & 5 & 0,57 & 0,544 \\
\hline Presencia de publicaciones del asesor & 6 & 3 & 0,28 & 0,187 \\
\hline
\end{tabular}


estudiantes ${ }^{(14)}$, debido probablemente a su ausencia dentro de los planes académicos, o a la deficiencia de políticas para su detección y penalización en las universidades ${ }^{(15)}$.

Si bien, no se encontró una asociación entre la presencia de plagio y de publicaciones de parte del asesor -debido al pequeño tamaño muestral-, el que éste publique, se comportaría como un factor protector. Asimismo, notamos que los asesores de 24 tesis no han publicado, lo que podría reflejar la ausencia de cultura de publicación ${ }^{(2)}$, o también que los asesores de tesis no investigan.

Adicionalmente, se puede apreciar, que los asesores no revisan en detalle los avances de las investigaciones que les presentan sus alumnos, ya que ellos en primera instancia pudieron encontrar el plagio, si es que hubiesen estado advertidos de que es una falta frecuente en estudiantes universitarios. Si bien no podemos generalizar estos resultados a otras universidades peruanas, pero si consideramos un estudio previo en otra universidad de Lima ${ }^{(9)}$, así como la experiencia de revistas peruanas estudiantiles ${ }^{(16)}$ y profesionales ${ }^{(13)}$, creemos que nuestros resultados ayudarán a las universidades a tener en cuenta que el plagio es frecuente entre sus estudiantes.

Por otro lado, los tipos de estudio más frecuentemente usados en las tesis analizadas fueron observacional, descriptivo, transversal y retrospectivo resultados que coinciden con Galan et al. ${ }^{(12)}$, quienes analizaron los trabajos presentados a los Congresos Científicos Nacionales de Estudiantes de Medicina, realizados entre 1993 y 2003, comunicando que los estudios transversales representaron el $58,4 \%$ y los descriptivos el $60,2 \%$. En nuestro estudio solamente una tesis fue del tipo experimental, dato que difiere del estudio anterior (12) en el que $17,4 \%$ de los estudios fueron experimentales. Estas observaciones coinciden con reportes que señalan que en Latinoamérica se realizan estudios descriptivos que pretenden ser la base para otros más elaborados (17). Asimismo, ninguno de los autores de las tesis había tenido experiencia previa de publicación, datos que contrastan con otras universidades públicas donde sus alumnos tienen publicaciones desde el pregrado ${ }^{(18,19)}$.

Consideramos una limitación del estudio no haber incluido la traducción de la introducción a otros idiomas para la búsqueda de plagio y haber realizado la búsqueda de tesis publicadas sólo seis meses después de su sustentación. Por otro lado, las tesis pueden tener más de un asesor que no es el oficial por parte de la universidad, por lo que ese aspecto no pudo ser evaluado; además no se revisó la sección de discusión de la tesis, donde posiblemente se hubiese encontrado mayor cantidad de párrafos con plagio.

Se concluye que el plagio es frecuente en las tesis para optar por el Título de Médico Cirujano en la universidad estudiada, y que es responsabilidad de las Facultades de Medicina tomar las medidas necesarias para evitarlo, tal y como se evidencia en nuestro estudio, el uso de el motor de búsqueda Google (www.google.com) es una herramienta útil en la detección de estas irregularidades éticas, por lo que recomendamos su uso.

\section{Fuente de Financiamiento}

Autofinanciado

\section{Conflictos de Interés}

PMT es editor científico de la Revista Peruana de Medicina Experimental y Salud Pública.

\section{REFERENCIAS BIBLIOGRÁFICAS}

1. Ramos-Rodríguez MI, Sotomayor R. Realizar o no una tesis: razones de estudiantes de medicina de una universidad pública y factores asociados. Rev Peru Med Exp Salud Publica. 2008; 25(3): 322-24.

2. Gutierrez C, Mayta P. Publicaciones desde el pregrado en Latinoamérica: Importancia, limitaciones y alternativas de solución. CIMEL 2003; 8(1): 55-60.

3. Bilic-Zulle L, Frkovic V, Turk T, Azman J, Petrovecki M. Prevalence of plagiarism among medical students. Croat Med J. 2005; 46(1): 126-31.

4. McCullough $\mathbf{M}$, Holmberg $\mathbf{M}$. Using the Google search engine to detect word for word plagiarism in master's theses: a preliminary study. Coll Stud J. 2005; 39(3): 435-41.

5. Rosselot E, Bravo M, Kottow M, Valenzuela C, O'Ryan M, Thambi S, et al. En referencia al plagio Intelectual. Documento de la Comisión de Ética de la Facultad de Medicina de la Universidad de Chile. Rev Med Chile. 2008; 136(5): 653-58.

6. Reyes H, Palma J, Andresen M. Ética de las publicaciones en revistas médicas. Rev Med Chile. 2007; 135(4): 52933

7. Otero J, Barrios I. Copiar, cortar y pegar en el desarrollo de investigaciones científicas. Rev Cub Salud Publica. 2007; 33(3): e16.

8. Rojas-Revoredo V, Huamaní C, Mayta-Tristán P. Plagio en publicaciones científicas en el pregrado: experiencias y recomendaciones. Rev Med Chile. 2007; 135(8): 1087-88.

9. Huamaní C, Dulanto-Pizzorni A, Rojas-Revoredo V. 'Copiar y pegar' en investigaciones en el pregrado: haciendo mal uso del Internet. An Fac Med (Lima). 2008; 69(2): 117-19.

10. Barrie JM, Presti DE. Digital plagiarism - The web giveth and the web shall taketh. J Med Internet Res. 2000; 2(1): e6. 
11. Weeks AD. Detecting plagiarism: google could be the way forward. BMJ. 2006; 333(7570): 706.

12. Galán E, Manrique N, Villavicencio E, Yllatopa E, Peralta M, De la Cruz W. Producción científica de los investigadores del pregrado de medicina humana del Perú 1993-2003. CIMEL 2005; 10(1): 41-48.

13. Mayta-Tristán P, Mezones-Holguín E. Aclaración editorial. Rev Peru Med Exp Salud Publica. 2009; 26(3): 411-12.

14. Huamaní C, Mayta-Tristán P, Rodríguez-Morales AJ. Irregularidades éticas en la investigación estudiantil. An Fac Med (Lima). 2008; 69(2): 146.

15. Ryan G, Bonanno H, Krass I, Scouller K, Smith L. Undergraduate and postgraduate pharmacy students'perceptions of plagiarism and academic honesty. Am J Pharm Educ. 2009; 73(6): 105.

16. Cabrera R, Mayo $C$, Suárez N, Infante $C$, Náquira $C$, García-Zapata M. Plagio en un artículo estudiantil. CIMEL. 2007; 12(1): 32-33.
17. Pellegrini Filho A, Goldbaum M, Silvi J. Producción de artículos científicos sobre salud en seis países de América Latina, 1973 a 1992. Rev Panam Salud Publica. 1997; 1(1): 23-34.

18. Huamaní C, Chávez-Solís $P$, Mayta-Tristán P. Aporte estudiantil en la publicación de artículos científicos en revistas médicas indizadas en SCIELO- Perú , 1997 2005. An Fac Med (Lima). 2008; 69(1): 42-45.

19. Huamaní C, Chavez-Solís P, Dominguez-Haro W, Solano-Aldana $\mathbf{M}$. Producción científica estudiantil: Análisis y expectativas. Rev Peru Med Exp Salud Publica. 2007; 24(4): 444-46.

Correspondencia: Jhan Carlos Saldaña Gastulo

Dirección: Los Geranios No 10, Miraflores, Castilla, Piura, Perú. Teléfono: (51 073) 343-131

Correo electrónico: jhancarlo_696@hotmail.com

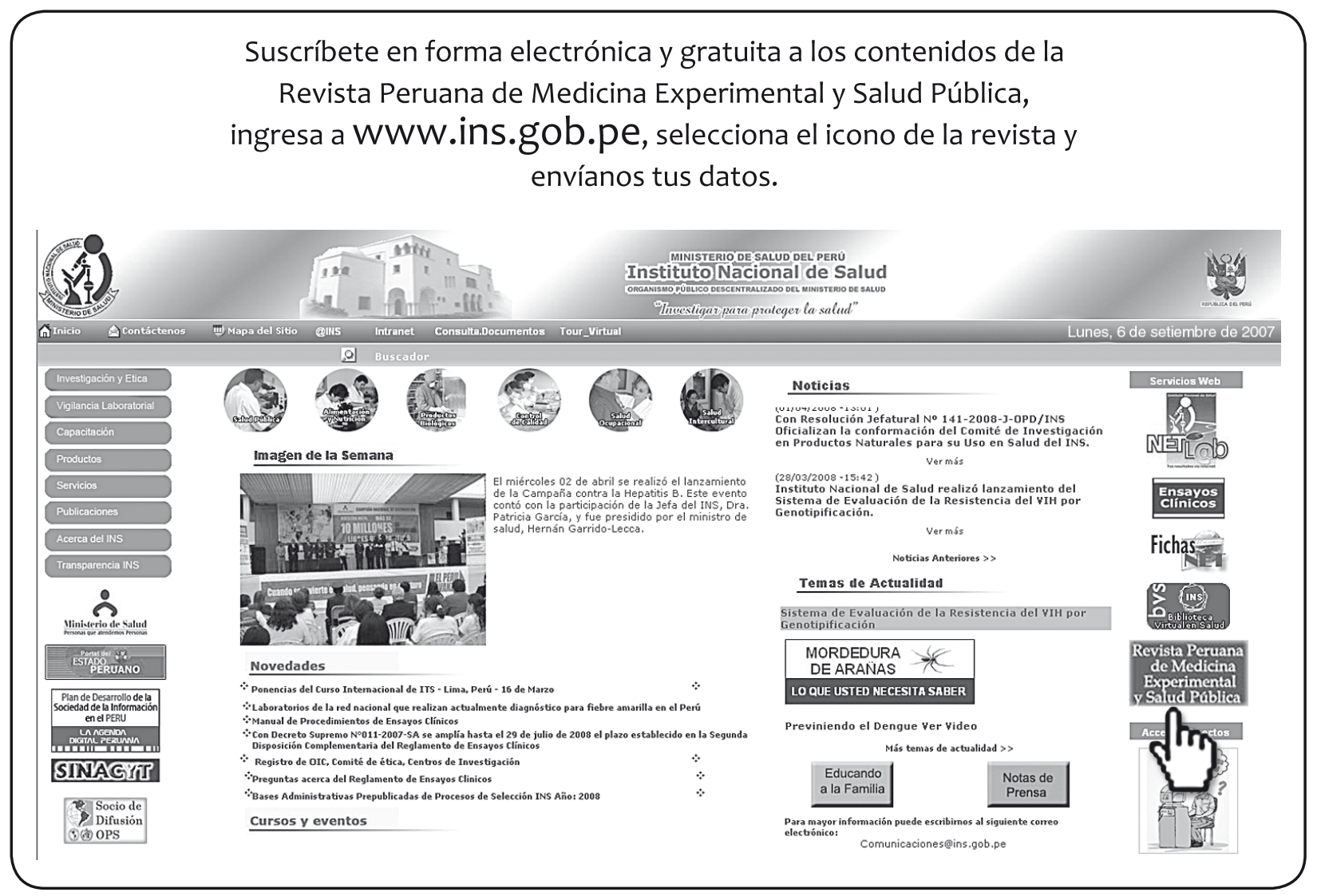

